Original

\title{
Cistoprostatectomia radical robótica: análisis oncológico y funcional
}

\author{
Joan Palou Redorta, Josep María Gaya Sopena, Lluís Gausa Gascón, Francisco Sánchez-Martín, \\ Antonio Rosales Bordes, Óscar Rodríguez Faba, Humberto Villavicencio Mavrich
}

\author{
Unidad de Urología Oncológica, Servicio de Urología, Fundació Puigvert, Universidad Autónoma de Barcelona, \\ Barcelona, España
}

\begin{abstract}
Resumen
Introducción: La cirugía laparoscópica robotizada da Vinci, ha demostrado en la prostatectomía radical, óptimos resultados funcionales y oncológicos con menor curva de aprendizaje, mayor comodidad y visión para el cirujano, y correcta preservación de las bandeletas neurovasculares. Esto ha dado lugar a que iniciar la experiencia con cistectomía radical robótica (CRR), fuera de nuestro país.

Objetivos: Revisar nuestra experiencia inicial en CRR, valorando los resultados quirúrgicos y funcionales obtenidos, y complicaciones inmediatas y a corto plazo.

Material y métodos: Entre diciembre del 2007 y enero del 2009 hemos realizado nueve cistoprostatectomías radicales robóticas y en siete de los nueve casos con linfadenectomía (LDN) robótica. En 5 pacientes por tumor músculo-invasivo y 4 por no músculoinvasivo de alto riesgo. La mediana de edad fue 57 años (rango 34-81). La derivación urinaria se realizó extracorpóreamente, en 3 casos un conducto ileal, en los 6 restantes una neovejiga tipo Studer, en 3 de estos 6 casos la anastomosis uretro-neovejiga se realizó intracorpóreamente.

Resultados: El tiempo medio total de cirugía fue 300 minutos (rango 280-420) en el conducto ileal y 360 (rango 330-540) en el Studer. Ningún caso requirió conversión ni transfusión sanguínea. La mediana de ganglios extraidos mediante LDN robótica fue 10 (rango 6-18). La AP de la pieza reveló 3 pT0, 2 CIS, 3 pT3, 1 pT4b (márgenes positivos). Con mediana de seguimiento de 7 meses no se ha observado ningún implante peritoneal y únicamente una estenosis ureteral. Se inició dieta oral en 5 casos a las 48 horas. De los 6 pacientes con función sexual conservada preoperatoria y seguimiento $>3$ meses, 2 tenían erección completa al mes, 2 a los 3 y 6 meses, y los 2 restantes presentan erección completa con inhib de la 5PDE a los 3 y 9 meses. Todos los pacientes con neovejiga presentan correcta continencia diurna. La estancia media hospitalaria fue de 8,5 días (rango 7-19).

Conclusiones: La cistectomía radical más linfadenectomía robótica, con reconstrucción extracorpórea de la derivación urinaria ofrece buenos resultados quirúrgicos y funcionales tempranos. La preservación cuidadosa de las bandeletas neurovasculares en esta cirugía radical pélvica permite obtener excelentes resultados en la función sexual y miccional.
\end{abstract}

Palabras clave: Cáncer de vejiga. Cistectomía radical. Laparoscopia. Linfadenectomía. Robot. Derivación urinaria.

Robotic radical cystoprostatectomy: oncological and functional analysis

\section{Abstract}

Introduction: The da Vinci robotic laparoscopic surgery, has been shown in radical prostatectomy, optimal functional and oncological results with a lower learning curve, greater comfort and vision for the surgeon, and proper preservation of the neurovascular bundles. This has led to begin the experience with robotic radical cystectomy (RRC).

Objectives: Review our initial experience in CRR, evaluating surgical and functional results obtained, and also immediate and short-term complications.

Material and methods: Between December 2007 and January 2009 we performed nine robotic radical cystoprostatectomy and in seven patients robotic lymphadenectomy (LDN). Five patients had a muscle-invasive disease and 4 non-muscle invasive bladder cancer. The median age was 57 years (range 34-81). Urinary diversion was performed extracorporeally in all cases, 3 cases an ileal conduit and 6 an Studer neobladder in 3 of these 6 cases, the urethra-neobladder anastomosis was performed intracorporeally.

Results: The average time of surgery was 300 minutes (range 280-420) in the ileal conduit and 360 (range 330-540) in the Studer. No cases required conversion or blood transfusion. The median number of nodes removed by LDN robotics was 10 (range 6-18). The pathology revealed 3 pTO, 2 CIS, 3 pT3, 1 pT4b (positive margins). With a median follow up of 7 months there have been no peritoneal implant and only one ureteral stenosis. Oral diet was initiated in 5 cases at 48 hours. Of the 6 patients with preserved sexual function preoperatively and followup of more than 3 months, 2 had full erection at 1 month, 2 at 3 and 6 months, and the remaining 2 presented with a full erection with 5 PD inhibitors at 3 and 9 months. All patients with neobladder presented correct daytime continence. The average hospital stay was 8.5 days (range $7-19$ ).

Conclusions: The radical robotic cystectomy with extracorporeal reconstruction of the urinary diversion offers good early functional and surgical outcomes. The careful preservation of the neurovascular bundles in radical pelvic surgery provides excellent results in urinary and sexual function.

Keywords: Bladder neoplasm. Laparoscopy. Lymphadenectomy. radical cystectomy. Robot. Urinary diversion. 
T a cistectomía radical (CR) abierta con linfadeLnectomía (LDN) ampliada es el tratamiento quirúrgico de elección para el tumor vesical músculo invasivo órgano-confinado y para el tumor superficial de alto riesgo que no responde a la BCG.

Sin embargo el desarrollo de las técnicas laparoscópicas y su aceptación para el tratamiento urooncológico del tumor renal y del cáncer de próstata ${ }^{1}$ ha permitido proponer la CR laparoscópica (CRL) como una alternativa a la cirugía abierta, en centros especializados. Desde los primeros casos de Parra ${ }^{2}$ y Sánchez-Badajoz ${ }^{3}$ todas las series publicadas coinciden en destacar las ventajas de la vía laparoscópica frente a la cirugía abierta en cuanto a disminución del sangrado, rápida recuperación y menor número de días de hospitalización, con resultados funcionales y oncológicos equiparables ${ }^{4,5}$.

La irrupción en el mercado del da Vinci Surgical System (Intuitive Surgical Inc, Sunnyvale, CA, USA) hizo que en el 2003 Menon $^{6}$ ya publicara la primera $\mathrm{CR}$ robótica. Tal como sucedió con la prostatectomía radical, la técnica robótica ha demostrado como mínimo los mismos resultados funcionales y oncológicos que el abordaje laparoscópico, con una menor curva de aprendizaje y mayor comodidad y visión para el cirujano ${ }^{7-9}$.

La expansión de esta nueva tecnología, sobre todo fuera de nuestro país, ha motivado diversas publicaciones donde se comunican los buenos resultados iniciales de la CR robótica ${ }^{10-12}$. En muchos de estos centros se ha ido sustituyendo la laparoscopia convencional por la robótica en casos de CR con LDN.
El objetivo de este artículo es presentar nuestra experiencia inicial en $\mathrm{CR}$ robótica (da Vinci), valorando los resultados quirúrgicos y funcionales (continencia miccional y potencia sexual), a la vez que los aspectos técnicos de esta nueva modalidad quirúrgica y las complicaciones inmediatas y a corto plazo.

\section{MATERIAL Y MÉTODOS}

Entre diciembre del 2007 y enero del 2009 se realizaron 9 casos de $\mathrm{CR}$ robótica. En todos los casos se trataba de varones, con una mediana de edad de 57 años (rango 34-81), índice masa corporal de 27,4 (rango 24,2-31,1) y mediana de seguimiento desde el momento de la cirugía radical de 7 meses (rango 1-13).

La indicación de CR correspondió a la presencia de carcinoma transicional músculo-invasivo en 5 casos, adenocarcinoma extenso en un paciente joven con lipomatosis pélvica, y carcinoma transicional no músculo-invasivo extenso o no respondedor a la BCG en otros 4 casos. (Tabla 1).

Ningún paciente había recibido quimioterapia previa y en todos los casos de tumor músculo-invasivo no pasaron más de 2 meses entre la RTU y la cirugía radical. Sólo un paciente tenía antecedentes de radioterapia pélvica por un adenocarcinoma de próstata. La exéresis completa de la vejiga se realizó con técnica robótica en todos los casos, añadiendo LDN robótica en 7 de ellos. En un caso no se realizó LDN (por extensa afectación extravesical y radioterapia pélvica) y en otro paciente afecto de lipomatosis pelviana la exéresis ganglionar se hizo vía abierta. Se

\section{Tabla 1}

\begin{tabular}{|c|c|c|c|c|c|}
\hline Casos & Edad & $\begin{array}{l}\text { Estadio clínico } \\
\text { (TNM) }\end{array}$ & $\begin{array}{l}\text { Estadio patológico } \\
\text { (pTNM) }\end{array}$ & $\mathbf{N}^{\circ}$ ganglios & $\begin{array}{l}\text { Derivación } \\
\text { urinaria }\end{array}$ \\
\hline 1 & 34 & Ca. cél. pequeña T2 & pTONO & 7 & C. ileal \\
\hline 2 & 61 & CIS múltiple & pTONO + CIS & 6 & Neovejiga Studer \\
\hline 3 & 44 & CU AG T1 múltiple & pT3NO & 10 & Neovejiga Studer \\
\hline 4 & 77 & CU AG T2 + CIS & pTONO + CIS & 7 & C. ileal \\
\hline 5 & 81 & $\begin{array}{l}\text { CU AG T3 (anaplásico) + } \\
\text { Adenoca. Próstata }\end{array}$ & $\begin{array}{l}\text { Cél. Pequeña pT4Nx } \\
\text { Adenoca. próstata }\end{array}$ & $\begin{array}{l}\text { No } \\
\text { LDN }\end{array}$ & C. ileal \\
\hline 6 & 59 & CU AG T1 múltiple & pTONO & 18 & Neovejiga Studer \\
\hline 7 & 48 & CU AG T2 & pT3NO & 10 & Neovejiga Studer \\
\hline 8 & 45 & Cist. glandular+Adenoca U. T1 & pTONO & $\begin{array}{l}6 \\
\text { (c.abierta) }\end{array}$ & Neovejiga Studer \\
\hline 9 & 63 & CU AG T2 & CU pT3aNO & 11 & Neovejiga Studer \\
\hline
\end{tabular}


practicó preservación de bandeletas neurovasculares a los 6 pacientes que presentaban función eréctil preoperatoria conservada. El extremo proximal de la uretra se cerró de forma sistemática con Hemo-lok antes de la sección uretral, para evitar implantación de células tumorales.

Las piezas una vez embolsadas se extrajeron por una incisión media infraumbilical de aproximadamente 6 centímetros de longitud por donde también se exteriorizó el ileon. El tiempo intestinal y la anastomosis ureteroileal se realizaron vía abierta en todos los casos. En todos los casos la anastomosis ureteroileal fue tipo Wallace II, con transposición retrosigmoidea del uréter izquierdo al lado derecho. En uno de los pacientes se realizó la transposición del uréter izquierdo y la confección de la placa de forma robótica, mediante sutura doble continua de monocryl 5/0, recurriéndose a la cirugía abierta con vicryl 4/0 para este tiempo quirúrgico en los otros 8 pacientes.

En 6 casos se confeccionó una neovejiga tipo Studer. En 3 de ellos la anastomosis uretro-neovejiga se realizó con sutura continua de forma intracorpórea, con la asistencia del robot. En los otros 3 casos se hizo una anastomosis manual clásica con 6 puntos de vicryl 2/0. La derivación urinaria de los restantes 3 pacientes restantes correspondió a conducto ileal tipo Bricker.

En los 9 casos se tutorizaron los uréteres con catéteres Argyle $7 \mathrm{Fr}$, exteriorizándose por el estoma en los casos de conducto ileal y por contrabertura a través de chimenea ileal en los casos de neovejiga, retirándose en la mayoría de los casos entre los siete y diez días de la intervención. En todos los casos se dejó drenaje abdominal. La sonda uretrovesical (Silicona, 20Fr) en los pacientes con neovejiga se retiró a los 15 días.

\section{TÉCNICA QUIRÚRGICA}

Con el paciente en posición de Trendelenburg $30^{\circ}$ con las piernas separadas, la izquierda más baja que la derecha, para permitir la entrada del plafón del robot provisto de cuatro brazos efectores (Fig.1), se realiza minilaparotomía media, dos centímetros por encima del ombligo y a 20 centímetros de la sínfisis púbica para colocación bajo visión directa del primer trocar de $12 \mathrm{~mm}$. Éste es un trocar convencional de laparoscopia, al que se adapta el brazo robótico que sostiene la cámara. Tras comprobación de que se ha penetrado en el espacio

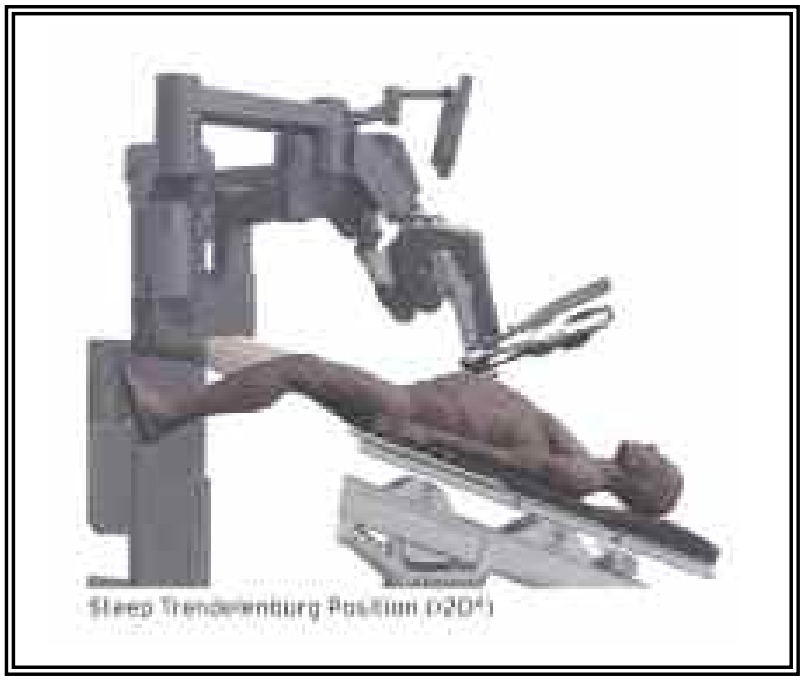

FIGURA 1. Colocación del paciente.

intraperitoneal, se induce el neumoperitoneo (flujo $25-30 \mathrm{ml} / \mathrm{min}$; presión a $13-15 \mathrm{mmHg}$ ) y se introducen el resto de trócares bajo visión laparoscópica.

La colocación de los puertos es clave para que no haya conflictos de espacio, como colisión entre brazos, y para que el instrumental llegue en todo momento a los puntos más distales del campo quirúrgico. Se colocan sucesivamente los 3 trócares robóticos de $8 \mathrm{~mm}$, los 2 primeros infraumbilicales, a derecha e izquierda, triangulando a $10 \mathrm{~cm}$ del trocar de la cámara y a $15 \mathrm{~cm}$ del pubis. El del lado derecho coincide con la marca en la piel prevista para el estoma. El tercer trocar robótico se emplaza a $2 \mathrm{~cm}$ por encima de la espina iliaca antero-superior izquierda, a $10 \mathrm{~cm}$ del trocar robótico del mismo lado. A continuación se colocan los trócares del cirujano ayudante (no robóticos); uno de $11 \mathrm{~mm}, 2$ $\mathrm{cm}$ por encima de la espina ilíaca antero-superior derecha, y otro de $5 \mathrm{~mm}$ a la altura del ombligo, entre el trocar de la cámara y el trocar robótico del lado derecho (Fig. 2).

Una vez colocados los puertos se insertan los anclajes de los brazos del robot al instrumental previamente colocado a través de los 3 trócares robóticos, el cirujano principal se desplaza a la consola para iniciar la CR. La disección robótica se inicia con una tijera monopolar curva y una pinza bipolar fenestrada (Maryland ${ }^{\mathrm{R}}$ ). En el cuarto brazo del robot se coloca un grasper que el cirujano utilizará para separar o sostener estructuras. El primer paso es la liberación de adherencias y la movilización del sigma, que puede quedar suspendido del cuarto brazo para facilitar la identificación del fondo de 


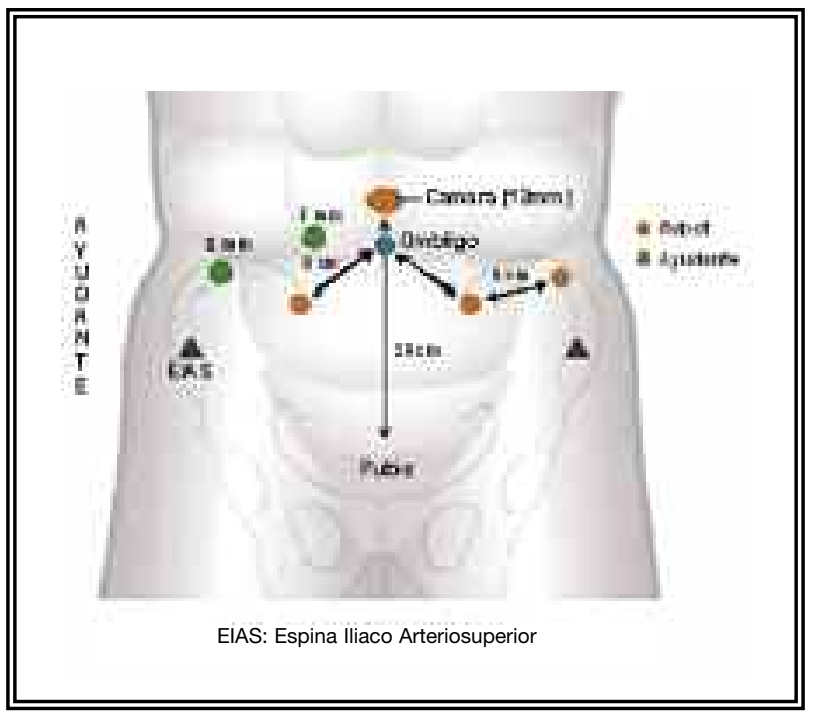

FIGURA 2. Disposición de los trócares.

saco de Douglas y la disección del uréter derecho. Se procede después a la apertura del fondo de saco de Douglas, y a la disección roma de la mitad de la cara posteriorvesicular a través del espacio rectovesical. Con electrocoagulación se diseca el uréter derecho hasta su entrada en vejiga. Sigue la disección del espacio laterovesical ipsilateral hasta identificar y abrir la fascia endopélvica. Se realiza el mismo procedimiento con el uréter del lado izquierdo. Luego se seccionan ambos uréteres a nivel distal con Hem-o-lok, para evitar salida de orina al campo operatorio. Una vez liberados los uréteres se procede a la liberación lateral de la vejiga con coagulación y maniobras de dirección roma. La visión tridimensional magnificada permite una elección correcta de los planos más adecuados. El ayudante secciona con Ligasure ${ }^{\mathrm{R}} 10 \mathrm{~mm}$ los conductos deferentes y los pedículos vesicales hasta llegar a identificar las vesículas seminales, que son apenas disecadas para que queden incluidas en el bloque del espécimen final. Se sigue con la apertura de la hoja posterior de la fascia de Denonvilliers y con la sección de los pedículos vesicoprostáticos y bandeletas con Ligasure ${ }^{\mathrm{R}}$ cuando el abordaje es extrafascial. Si se desea preservar las bandeletas neurovasculares, se practica una disección intrafascial que permite un buen control de los pedículos vesicoprostáticos con Hem-olocks, con mínima electrocoagulación, hasta llegar a la cara posterior del ápex prostático. Una vez finalizada la disección de la cara posterior de la pieza de $\mathrm{CR}$, se procede a la sección del peritoneo parietal anterior a nivel del uraco para conseguir el descenso de la vejiga y llegar al espacio de Retzius. Tras la sección de ligamentos puboprostáticos, se liga y se secciona el complejo venoso dorsal, igual que en la prostatectomía radical, y se procede a la disección de las distintas caras de la uretra. La lenta retirada de la sonda vesical permite la sección de la uretra con Hem-o-lok, lo más lejos posible del ápex prostático pero dejando un buen muñón uretral cuando sea necesaria una anastomosis a neovejiga. El empleo de Hem-o-lok impide la fuga de células neoplásicas al lecho quirúrgico. Una vez liberada la pieza, se embolsa en un Endocatch $^{\mathrm{R}}$ de $15 \mathrm{~mm}$, que se introduce ampliando el trocar de $11 \mathrm{~mm}$ del ayudante, evitando así colocar un nuevo trocar. Tras el cierre de la bolsa se exterioriza parcialmente por el orificio del trocar y, con la ayuda de un grasper, se recoloca de nuevo el trocar de $11 \mathrm{~mm}$. Esta maniobra permite que el borde de la bolsa se pueda cerrar con un Kocher y quede aislado de la zona de trabajo. A la vez permite que el ayudante mantenga el puerto de $11 \mathrm{~mm}$, necesario para la LDN.

La LDN se realiza después de la CR. Los múltiples ejes de giro del instrumental robótico facilitan la disección craneal de los vasos ilíacos, sin quedar afectada por la localización infraumbilical de los puertos. Una vez terminada la LDN bilateral se embolsan las muestras por separado en sendos Endocatch $^{\mathrm{R}}$ de $5 \mathrm{~mm}$.

Antes de pasar al tiempo abierto se revisa la hemostasia, comprobando que la disección craneal de los uréteres sea suficiente para no tener que ampliar después la incisión abdominal. Se desacoplan los brazos del robot, sin perder la esterilidad, y se mantienen los trócares colocados, aunque retirados parcialmente. De esta manera será posible, más adelante, realizar la anastomosis uretroneovesical de forma intracorpórea en aquellos casos en que sea necesario.

El siguiente tiempo es la realización de una laparotomía media infraumbilical de unos 6 centímetros, por donde se extraen las piezas embolsadas previamente. A continuación se coloca un separador autoestático Bookwalter ${ }^{\mathrm{R}}$ para optimizar el campo profundo a través de la pequeña incisión cutánea. Se realiza la transposición del uréter izquierdo a través del mesosigma y se confecciona la placa del Wallace II. Se aísla un segmento ileal de 15 ó $55 \mathrm{~cm}$, según sea la derivación urinaria a realizar y se 
reconstruye el tránsito intestinal con sutura mecánica. Si se confecciona un conducto ileal se procede a la anastomosis ureteroileal, exteriorizando el extremo distal del ileon a través del orificio del trocar robótico derecho y se confecciona el estoma. Si se opta por una neovejiga tipo Studer se realiza la anastomosis uretroneovejiga en el punto más declive de la bolsa, bien a mano con 6 puntos sueltos de vicryl $2 / 0$ o bien intracorpórea con la ayuda del robot. En este caso, antes de cerrar la laparotomía media e inducir de nuevo el neumoperitoneo, se deben exteriorizar por contrabertura los catéteres ureterales, sin fijarlos a piel. También es el momento de colocar un punto próximo a la apertura hecha en la parte más declive de la neovejiga que permitirá traccionar de la bolsa, facilitando la sutura, si se opta por una anastomosis intracorpórea.

En este último supuesto, corresponde seguir con cierre de la pared abdominal (sutura continua de PDS) y piel con grapas. Se induce de nuevo el neumoperitoneo, comprobando la ausencia de fugas, y se acopla de nuevo el robot a los trócares, aunque con menos inclinación de la mesa operatoria para facilitar el descenso de la neovejiga hacia la pelvis. $\mathrm{El}$ ayudante desplaza la neovejiga con 2 graspers hasta llegar a contactar con la uretra y el cirujano acciona el cuarto brazo del robot hasta fijar en la posición deseada el punto de referencia colocado antes. Una vez enfrentados los bordes a suturar mediante los trócares robóticos se realiza una anastomosis continua con monocryl 5/0. Se coloca sonda siliconada $20 \mathrm{Fr}$ y, tras comprobar la estanqueidad de la sutura y la corrección de la hemostasia, se deja drenaje que se introduce por uno de los trócares. Se retira los brazos del robot y los trócares bajo visión laparoscópica, suturando los de más de $10 \mathrm{~mm}$.

\section{RESULTADOS}

Tiempo operatorio: El tiempo medio empleado para los diversos tiempos de la intervención robótica fue de 140 minutos (rango 90-180) para la CR y de 60 minutos (rango 45-90) para la LDN. Si se añade el tiempo empleado en la derivación urinaria, resulta una duración media total de 300 minutos (rango 280-420) para la opción conducto ileal y de 360 minutos (rango 330-540) para la neovejiga ortotópica tipo Studer. La mediana de sangrado perioperatorio fue de $325 \mathrm{~mL}$. Ningún paciente requirió transfusión sanguínea intraoperatoria.
Postoperatorio: La sonda nasogástrica se retiró antes de las 24 horas en 7 pacientes y a las 48 horas en 2. Se inició la tolerancia oral a las 48 horas en 5 casos y a las 72 horas en 4 . Dos pacientes presentaron íleo paralítico que retrasó la ingesta oral hasta el sexto y séptimo día respectivamente. Los catéteres ureterales se retiraron a los 7 días y el drenaje abdominal al octavo día en todos los casos. La estancia hospitalaria media fue de 8,5 días (rango 719), siendo superior en los portadores de neovejiga.

Resultado patológico: Los estadios patológicos de los especímenes extirpados están resumidos en la tabla 1. La mediana de ganglios extraídos mediante LDN robótica fue de 10 (rango 6-18), siendo todos ellos negativos. El margen quirúrgico fue positivo en un único caso (carcinoma avanzado localmente, con antecedentes de radioterapia prostática).

Complicaciones postoperatorias inmediatas: Hematoma localizado de pared abdominal por sangrado de trayecto del trocar en 1 paciente. Infección de la herida quirúrgica en 1 paciente. Ambas complicaciones se resolvieron con tratamiento conservador.

Complicaciones a medio plazo: Estenosis ureteral distal izquierda en 1 caso de neovejiga ortotópica, siendo necesaria la colocación de una nefrostomía por punción percutánea y posterior reimplante ureteral vía abierta. Infección urinaria febril en otro paciente con neovejiga, resuelta con tratamiento médico.

Resultados oncológicos: Todos los pacientes están libres de enfermedad. Tres pacientes ( 2 pT3 y 1 pT4) recibieron quimioterapia adyuvante.

Resultados funcionales: Los 6 pacientes con neovejiga presentan una correcta continencia, sin necesidad de protección, diurna y nocturna. De los 6 pacientes con función sexual previa normal (a todos ellos se les realizó conservación de bandeletas), 2 tenían erección completa espontánea al mes de la intervención y 2 la adquirieron entre $\operatorname{los} 3$ y 6 meses. Los 2 restantes han conseguido erección completa con inhibidores de la 5-PDE, a partir de los 3 y 9 meses respectivamente.

\section{DISCUSIÓN}

La CR con LDN robótica es una técnica reciente, reservada para aquellos centros que han incorporado el robot da Vinci y han adquirido experiencia en cirugía asistida por robot. Son más de 350 los casos realizados en todo el mundo ${ }^{13}$, la mayoría de ellos en centros hospitalarios de los EEUU. La velocidad 
de implantación ha sido más rápida que la que tuvo la CR laparoscópica, debido a la experiencia adquirida con la prostatectomía radical robótica, verdadera precursora de las demás técnicas.

La ergonomía y la definición visual (aumento, tridimensionalidad) aportan a la cirugía robótica ventajas sobre la laparoscopia convencional. La robótica mantiene la mayoria de ventajas que la laparoscopia presenta sobre la cirugía abierta, como son la menor invasividad y más rápida recuperación del paciente. Los costes brutos son mejores para la laparoscopia. Las series publicadas que comparan la CR laparoscópica (CRLap) con derivación urinaria extracorpórea con la $\mathrm{CR}$ abierta refieren que aquélla presenta menor sangrado, con pérdidas de entre 300-400mL; menor dolor postoperatorio y menor requerimiento analgésico; disminución de la estancia hospitalaria y de la convalescencia; y recuperación del tránsito intestinal y de la tolerancia oral más precoz, por el menor tiempo de exposición intestinal ${ }^{14-16}$. Estas series, publicadas por laparoscopistas expertos no encuentran diferencias significativas entre los tiempos quirúrgicos de ambas técnicas, pero hay que recordar que los primeros casos descritos de CRLap eran muy largos, debido a la prolija curva de aprendizaje de la laparoscopia. Una de las ventajas clave de la cirugía robótica es su corta curva de aprendizaje, lo que permite alcanzar tiempos operatorios comparables a los de la cirugía abierta ya desde los primeros $\operatorname{casos}^{6,17,18}$.

Las mejoras en visión del robot permite una disección más cuidadosa, implicando un mejor control de la hemostasia, situando la necesidad de transfusión intraoperatoria por debajo del $<5 \%$. El confort del cirujano durante la cistoprostatectomía robótica puede traducirse en una menor tasa de complicaciones y unos resultados oncológicos y funcionales mejores, si bien estas conclusiones requieren de estudios futuros.

La técnica de la CR robótica con su correspondiente derivación urinaria está aún en evolución; por lo tanto no existe una técnica universalmente aceptada. Los pasos clave de la intervención se basan en la experiencia anterior con la CR abierta y la laparoscópica, y son comunes en los urólogos que están realizando cirugía robótica. Como sucedió con la laparoscopia convencional ${ }^{19}$, algunos autores han publicado su experiencia robótica en la construcción intracorpórea de la derivación urinaria ${ }^{20-23}$. La revisión de la Cleveland Clinic ${ }^{24}$ compara 17 pacientes sometidos a CRLap con construcción completa intracorpórea de la derivación urinaria, con otros 37 donde la derivación urinaria se realizó de forma extracorpórea, demostrando que si se realiza el tiempo intestinal en modo abierto se reduce el tiempo quirúrgico y disminuye el número de complicaciones graves.

Los tiempos medios quirúrgicos publicados, con la realización extracorpórea de la derivación urinaria, son de entre 160-200 minutos para la parte exerética más 130-150 minutos para un conducto ileal o 180-200 minutos para una vejiga ortotópica ${ }^{6,11,18}$, resultando en un tiempo quirúrgico total de entre 58 horas, similares a los obtenidos en nuestra serie.

Con cirugía robótica los límites quirúrgicos de la LDN deben ser los mismos que en la cirugía abierta. La extensión de la LDN ha demostrado tener valor pronóstico y terapéutico, por lo que se aconseja una exéresis ganglionar amplia, con un mínimo de 1014 ganglios extraídos ${ }^{25-28}$. Aunque en nuestra serie inicial, el número es menor, hemos ido aumentando progresivamente el número de ganglios extraídos. Algunos laparoscopistas abogan por realizar la LDN al inicio de la cirugía, tras la disección ureteral. Se facilita, según ellos, la posterior disección de la cara lateral de la vejiga y la ligadura y sección de los pedículos laterales ${ }^{29}$. Somos partidarios del Ligasure $^{\mathrm{R}} 10 \mathrm{~mm}$ para realizar esta maniobra, que consigue una rápida y segura hemostasia.

En cirugía robótica la mayoría de autores prefiere realizar LDN tras haber embolsado la pieza de $\mathrm{CR}$, opinión con la que coincidimos. Las series publicadas hasta la fecha de CR robótica no han informado de manera uniforme de la extensión de la LDN pélvica realizada o del número de ganglios linfáticos extirpados. Sólo Menon en su serie de 17 casos habla de LDN extendida con un rango entre 4 y 27 ganglios 6 .

Menon et al. describieron la CR robótica con preservación neurovascular ${ }^{6}$ con objeto de mejorar los resultados funcionales. Las claves de esta técnica fueron la preservación de la arteria capsular y la utilización de las vesículas seminales como punto de referencia quirúrgica. Sin embargo, los resultados publicados (14 varones) no contenían datos sobre la potencia. En cirugía abierta y laparoscópica es posible la preservación si se emplea una técnica depurada, obteniendo buenos resultados funcionales ${ }^{30}$. Nuestra experiencia con la preservación de bandeletas neurovasculares en la prostatectomía radical robótica ${ }^{8}$ ha 
permitido progresar más rápidamente en este aspecto al iniciar la cirugía radical vesical obteniendo los buenos resultados presentados. En el grupo de preservación neurovascular (6 pacientes), el 100\% presentan relaciones sexuales satisfactorias tras una media de 7 meses de seguimiento, con erección completa, aunque 2 precisan de tratamiento médico con inhibidores de la 5 fosfodiesterasa. El empleo de estos fármacos es opcional para los pacientes que no muestran una adecuada respuesta eréctil precoz. En lo referente a mujeres, los datos relativos al efecto de la $\mathrm{CR}$ en cuanto a la función sexual son escasos. Menon et $\mathrm{al}^{31}$ describieron la preservación del útero y la vagina en 3 mujeres que se sometieron a CR robótica. Se esperan los resultados funcionales y oncológicos de estas pacientes.

Una de las limitaciones actuales de las series de CR robótica es la escasez de resultados oncológicos a largo plazo. Es previsible que sean similares a los que se pueden obtener con la CRLap, si bien son pocas las series publicadas con resultados oncológicos a 5 años que permitan corroborarlo. Recientemente el grupo de Cleveland Clinic ha revisado sus resultados de 37 CRLap. Se realizó LDN pélvica extendida en 26 de los pacientes (70\%). El promedio de ganglios linfáticos extirpados fue de 14 . Ocho pacientes (22\%) han completado un seguimiento de 5 años. Dos presentaron margen quirúrgico positivo. La supervivencia global y cáncer-específica fue de 63 y 92\%, respectivamente. Deger et al. ${ }^{32}$ publicaron sus resultados con 20 CRLap y reservorio rectosigmoideo intracorpóreo (seguimiento medio de 33 meses). Todos los márgenes quirúrgicos fueron negativos, pero 3 pacientes tenian ganglios positivos. Durante el seguimiento 3 pacientes desarrollaron metástasis y 2 fallecieron por cáncer a los 15 y 24 meses siguientes a la cirugía. El consenso internacional en la $\mathrm{CR}$ robótica es que la derivación urinaria debe realizarse vía abierta ya que la realización intracorpórea alarga el tiempo quirúrgico y aumenta el número de complicaciones. Hasta hoy sólo se ha informado de un caso de metástasis en los puertos de entrada después de CR robótica en un caso de tumor vesical músculoinvasivo y de alto $\operatorname{grado}^{33}$.

El futuro de la cirugía radical oncológica vesical consiste en consolidar técnicas cada vez menos agresivas que garanticen unos buenos resultados oncológicos y funcionales, en especial los aspectos miccional y sexual, siguiendo criterios similares a los empleados para evaluar las diversas modalida- des de prostatectomía radical ${ }^{34}$. En lugar preferente figura el cumplimiento de los criterios oncológicos. En laparoscopia convencional se han demostrado resultados similares a la cirugía abierta, pero en cirugía robótica es necesario seguimiento a más largo plazo. A continuación figuran los resultados funcionales. Conseguir una buena función miccional, ofreciendo, siempre que sea posible, una neovejiga ortotópica (66,6 \% de nuestra serie robótica) es deseable. Por último, lograr el mantenimiento de una correcta función sexual (100\% en pacientes con conservación de bandeletas en nuestra serie robótica), es un objetivo importante para la calidad de vida de los pacientes. Las ventajas en aspectos como el dolor postoperatorio, transfusión, recuperación intestinal y posibilidad de un alta hospitalaria precoz, son puntos a favor de la cirugía radical vesical robótica. Es necesario no obstante consolidar los resultados con seguimientos más largos.

Los resultados oncológicos y funcionales a largo plazo, así como los temas de costes son importantes para definir el papel exacto de la CR mínimamente invasiva robotizada en el manejo del cáncer de vejiga.

\section{CONCLUSIONES}

La CR robótica más $\mathrm{LDN}$, con reconstrucción extracorpórea de la derivación urinaria, combina las ventajas de una cirugía mínimamente invasiva, con la seguridad y los resultados oncológicos de la cirugía abierta. Frente a ésta, la cirugía robótica presenta, al igual que la laparoscopia clásica, ventajas en aspectos como el sangrado intraoperatorio, dolor postoperatorio, recuperación del tránsito intestinal y de la tolerancia oral, así como menor estancia hospitalaria. Una ventaja de la robótica respecto a la laparoscopia es que precisa de una menor curva de aprendizaje, con tiempos operatorios competitivos desde los primeros casos. La cirugía robótica aporta también una mayor calidad de visión y gestualidad para el cirujano, con mejores condiciones ergonómicas, lo que permite realizar la intervención reduciendo la fatiga y mejorando la precisión de movimientos, todo lo cual puede traducirse en mejores resultados. Desde el punto de vista oncológico los resultados a medio plazo de la CR laparoscópica son satisfactorios y comparables con las series de cirugía abierta, en la robótica aun se dispone de un escaso seguimiento. Desde el punto de vista funcional, permite una excelente calidad miccional y sexual en los casos que se preservan las bandeletas neurovasculares. 


\section{REFERENCIAS}

1. Guillonneau B, el-Fettouh H, Baumert H, Cathelineau X, Doublet JD, Fromont G, et al: Laparoscopic radical prostatectomy:oncological evaluation after 1000 cases a Montsouris Institute. J Urol 2003;169(4):1261-1266.

2. Parra RO, Andrus CH, Jones JP, Boullier JA. Laparoscopic cystectomy: initial report for a new treatment for the retained bladder. J Urol 1992;148(4):1140-1144.

3. Sánchez de Badajoz E, Gallego JL, Reche A, Gutíerrez de la Cruz JM, Jiménez A. CR radical y conducto ileal laparoscópico. Arch Esp Urol. 1993;46(7):621-624.

4. Haber GP, Campbell SC, Colombo JR, et al. Comparasion between open and laparoscopic assisted radical cystectomy for bladder cancer. J Urol 2007;177:548.

5. Porpiglia F, Renard J, Billia M, Scoffone C, Cracco C, Terrone $\mathrm{C}$ et al. Open vs laparoscopy-assisted radical cystectomy: results of a prospective study. J Endourol 2007;21(3):325-329.

6. Menon M, Hemal AK, Tewari A, Shrivastava A, Shoma AM, ElTabey NA et al. Nerve-sparing robot-assisted radical cystoprostatectomy and urinary diversion. BJU Int 2003;92(3):232-236.

7. Mattei A, Naspro R, Annino F, Burke D, Guida R Jr, Gasron R. Tension and energy-free robotic-assisted laparoscopic radical prostatectomy with interfascial dissection of the neurovasculars bundles. Eur Urol 2007;52(3):687-694.

8. Villavicencio H, Esquena S, Salvador J, Gómez-Ruiz JJ, Escovar La Riva P, Palou J. Prostatectomía radical robótica: análisis después de 100 casos en la Fundació Puigvert. Act Urol Esp. 2009, en prensa.

9. Badani KK, Kaul S, Menon M. Evolution of robotic radical prostatectomy: assessment after 2766 procedures. Cancer 2007. 110(9): 1951-1958.

10. Hemal AK, Abol-Enein H, Tewari A, Shrivastava A, Shoma AM, Ghoneim MA et al. Robotic radical cystectomy and urinary diversion in the management of bladder cancer. Urol Clin North AM 2004;31(4 special issue):719-729.

11. Pruthi RS, Wallen EM. Robotic-assisted laparoscopic radical cystoprostatectomy. Eur Urol 2008;53(2):310-322.

12. Wang GJ, Barocas DA, Raman JD, et al. Robotic vs.open radical cystectomy: prospective comparasion of perioperative outcomes and pathological measures of early oncological efficacy. BJU Int 2008;101(1):89-93.

13. Hemal AK. Role of robot-assisted surgery for bladder cancer. Curr Opin Urol 2009;19(1):69-75.

14. Porpiglia F, Renard J, Billia M, Scoffone C, Cracco C, Terrone $\mathrm{C}$ et al. Open versus laparoscopy-assisted radical cystectomy: results of a prospective study. J Endourol 2007;21(3):325-329.

15. Basillote JB, Abdelsheid C, Ahlering TE, Shanberg AM. Laparoscopic assisted radical cystectomy with ileal neobladder: a comparasion with the open approach. J Urol 2004;172(2): 489-493.

16. Cecchini L, Orsola A, Raventos C, Trilla E, Morote J. Cistectomía radical laparoscópica en tumors vesicales clínicamente localizados (T2). Arch. Esp. Urol. v.61 n.4. Mayo 2008.

17. Galich A, Sterrett S, Nazemi T, Pohlman G, Smith L, Balaji KC. Comparative analysis of early perioperative outcomes following radical cystectomy by either the robotic or open method. JSLS 2006;10(2): 145-150.

18. Beecken WD, Wolfram M, Engl T, Bentas W, Probst M, Blaheta $\mathrm{R}$ et al. Robotic-assisted laparoscopic radical cystectomy and intra-abdominal formation of an orthotopic ileal neobladder. Eur Urol 44(3) 337-339.

19. Alonso y Gregorio S, Alvarez Maestro M, Cabrera Castillo PM, Tabernero Gómez A, Cansino Alcaide R, Cisneros Ledo J et al. Derivaciones urinarias laparoscópicas. Actas Urol Esp. 2008;32(9):908-915
20. Yohannes P, Puri V, Yi B, Khan AK, Sudan R. Laparoscopyassisted robotic radical cystoprostatectomy with ileal conduit urinary diversion for muscle-invasive bladder cancer: initial two cases. J Endourol 2003;17(9):729-732.

21. Balaji KC, Yohannes P, McBride CL, Oleynikov D, Hemstreet GP 3rd Feasibility of robot-assisted totally intracorporeal laparoscopic ileal conduit urinary diversion: initial results of a single institutional pilot study. Urology 2004;63(1):51-55.

22. Hubert J, Chammas M, Larre S, Feuillu B, Cheng F, Beis JM, Initial experience with successful totally robotic laparoscopic cystoprostatectomy and ileal conduit construction in tetraplegic patients: report of two cases. J Endourol 2006;20(2):139143

23. Sala LG, Matsunaga GS, Corica FA, Ornstein DK. Robot-assisted laparoscopic radical cystoprostatectomy and totally intracorporeal ileal neobladder. J Endourol 2006;20(4):233-235.

24. Haber GP, Crouzet S, Gill IS. Laparoscopic and Robotic Assisted Radical Cystectomy for Bladder Cancer: A Critical Analysis. Eur Urol 2008;54(1):54-62.

25. Konety BR, Josyln SA, O'Donell MA. .Extent of pelvic lymphadenectomy and its impact on outcome in patients diagnosed with bladder cancer: analysis of data from the Surveillance, Epidemiology and End Results Program database. J Urol 2003 169(3):946-950.

26. Kassouf W, Leibovidici D, Munsell MF, Dinney CP, Kamat AM. Evaluation of the relevance of lymph node density in a contemporany series of patients undergoing radical cystectomy. $\mathrm{J}$ Urol 2006:176(1):53-57.

27. Herr HW. Superiority of ratio based lymph nodestaging for bladder cancer. J Urol 2003; 169(3):943-45.

28. Monzó JI, Herranz Amo F, Cabello Benavente R, Hernández Fernández C. Utilidad de la linfadenectomía en el cáncer de vejiga. Revisión de la literatura. Actas Urol Esp. 2007; 31(1): 1-6.

29. Castillo O, Cabello R, Briones G, Hernández C. CR radical laparoscópica. Actas Urol Esp 2006; 30(5):531-40.

30. Hekal IA, El-Bahnasawy MS, Moshah A, El-Assmy A, Shaaben A. Recoverability of erectile function in post-radical cystectomy patients: subjective and objective evaluation. Eur Urol 2008;53(6): 1193

31. Menon M, Hemal AK, Tewari A, Shrivastava A, Shoma AM, Abol-Ein H. Robot-assisted radical cystectomy and urinary diversion in female patients: technique with preservation of the uterus and vagina. J Am Coll Surg 2004 Mar;198(3):386-393.

32. Deger S, Peters R, Roigas J. Laparoscopic radical cystectomy with continent urinary diversion (rectosigmoid pouch) performed completely intracorporeally: an intermediate functional and oncologic analysis.Urology 2004;64(5):935-939.

33. El-Tabey NA, Shoma AM. Port site metastases after robotassisted laparoscopic radical cystectomy. Urology 2005;66(5): 1110 .

34. Pierorazio PM, Spencer BA, McCann TR, McKiernan JM, Benson MC. Preoperative risk stratification predicts likelihood of concurrent PSA-free survival, continence, and potency (the trifecta analysis) after radical retropubic prostatectomy. Urology 2007;70:717-722.

Correspondencia autor: Dr. Joan Palou Redorta

Servicio de Urología. Fundació Puigvert

Cartagena 340-350 - 08025 Barcelona

Tel.: 934169700

E-mail autor: jpalou@fundacio-puigvert.es

Información artículo: Original - Laparoscopia

Trabajo recibido: marzo 2009

Trabajo aceptado: abril 2009 